\title{
Endoscopic approach of sinonasal inverted papilloma - our 15 years' experience on 162 cases
}

\author{
Vlad Budu', Alexandra Schnaider', loan Bulescu ${ }^{2}$ \\ 1"Prof. Dr. Dorin Hociota” Institute of Phono-Audiology and Functional ENT Surgery, Bucharest, \\ Romania \\ ${ }^{2}$ Department of Anatomy, "Carol Davila” University of Medicine and Pharmacy, Bucharest, Romania
}

\begin{abstract}
BACKGROUND. Inverted papilloma is a benign tumor of the nose and sinuses, with a high risk of recurrence and malignant degeneration. The inverted papilloma is a slow growing tumor that can be approached through an endoscopic or external approach, depending on its stage.

OBJECTIVE. The aims of the study are to identify the particularities of diagnosis of the inverted papilloma, to establish the correct steps in surgical treatment of this tumor and to open the access for other steps of treatment.

MATERIAL AND METHODS. The authors present their experience in managing the sinonasal inverted papilloma in a 15-year retrospective clinical study, which included 162 patients. The preoperative protocol consisted in clinical examination, nasal endoscopy, radiologic imaging (CT scan) and biopsy with histopathology results and immunohistochemistry findings. Surgical removal of the inverted papilloma was performed by endoscopic techniques, according to the stage of the tumor. We were interested in the recurrence rate of the tumor and its malignancy after a long-term follow-up.

RESULTS. In our series, we included 162 patients and we had 26 (16.04\%) recurrences and $12(7.40 \%)$ malignant degenerations. All our patients were diagnosed in Krouse stages I, II and III and underwent endoscopic resection of the tumor.

CONCLUSION. In order not to have any leftover tumor (the most important factor of recurrence and malignant transformation), it is mandatory to have a complete diagnosis of the inverted papilloma, a precise surgical technique and a rigorous followup. In some cases, the surgical treatment is associated with other type of treatment (antiviral, antiangiogenetic).
\end{abstract}

KEYWORDS: inverted papilloma, sinonasal papilloma, endoscopic sinus surgery, benign sinonasal tumors.

\section{INTRODUCTION}

The sinonasal papilloma is a benign neoplasm of the epithelium from the nasal cavity and sinuses. Inverted papilloma (IP), also known as Schneiderian papilloma, Ewing's tumor or transitional cell papilloma, usually arises from the lateral nasal wall ${ }^{1-3}$ into the middle meatus with local extension to the paranasal sinuses and neighbouring structures. Isolated involvement of the sphenoid sinus has also been reported by some authors ${ }^{2,3}$. Sinonasal papilloma is almost always unilateral and originates from a narrow or broad-based stalk. Clinical presentation of inverted papilloma depends on the sites of involvement, but the most common symptom is progressive unilateral nasal obstruction ${ }^{1,4,5}$.

The etiology of inverted papilloma is uncertain, but there are a lot of factors involved (allergy, tobacco, air pollution, environmental carcinogens).

Nowadays, the viral etiology of inverted papilloma is much more accepted and confirmed in lab (in situ DNA hybridization). Studies that used in situ hybridization and polymerase chain reaction (PCR) have detected human papilloma virus (HPV) in up to $86 \%$ of inverted papillomas $^{6,7}$. Human Papilloma Virus (HPV) is a virus with epithelial tropism, which is implicated in premalignant and malignant lesions (congenital tract), but into the nasosinusal region, there were described low risk 
subtypes (HPV 6, HPV 11) and high risk subtypes (HPV 16, HPV 18). The presence of HPV DNA in inverted papilloma has been found to be associated with higher chance of recurrence and malignant transformation ${ }^{1,6,7}$.

The histological findings of the inverted papilloma show an endophytic or inverted growth pattern, with the thickened squamous epithelium admixed with mucocytes "inversing" into the adjacent conjunctival stroma ${ }^{8}$.

Physical examination reveals a unilateral polypoid mass, which causes unilateral nasal obstruction. The tumor is reddish grey in color, with an irregular friable appearance and often bleeds when touched. Other symptoms that may occur include epistaxis, nasal discharge, epiphora and facial pain.

In order to have a correct diagnosis of the tumor, after nasal endoscopy, it is mandatory to have a preoperative imaging assessment by computed tomography (CT) or magnetic resonance imaging (MRI).

The CT scan, using both coronal and axial views, is considered the "gold standard" investigation before endoscopic sinus surgery in order to see the involvement of the adjacent bony structures (medial maxillary wall, lamina papyracea, skull base, pterygopalatine fossa). CT scans can determine the locations of the areas of focal hyperostosis, which in $89.1 \%$ of cases correspond to the actual origin of the tumor ${ }^{9,10}$.

MRI is an alternative imaging method for inverted papilloma in high stages, helpful due to its capacity to better differentiate soft tissue between each other. The typical aspect of IP on MRI scans shows a columnar pattern with a cerebriform aspect. This aspect has a positive predictive factor of $95.8 \%$ for $\mathrm{IP}^{1,11,12}$.

IP have a great destructive capacity on surrounding structures, a tendency to reoccur and a risk for malignant transformation.
The treatment of inverted papilloma of the nose and paranasal sinuses remains a clinical challenge to the ENT surgeon. Traditional treatment of inverted papilloma is surgical removal, and various approaches have been recommended over the years (external and endoscopic approaches).

The endoscopic removal of the inverted papilloma can be performed in stages I, II and in most cases of stage III and it should be based on the location and extent of the tumor (Table 1).

\section{MATERIAL AND METHODS}

In this long-term study, we were interested in identifying the particularities of endoscopic examination, in indentifying the particularities of the CT findings in inverted papilloma and in choosing the correct endoscopic surgical technique adapted to the stage of the tumor. We wanted to compare our recurrence rate with literature and to compare our malignancy rate on patients with inverted papilloma for a 15-year period.

Usually, the endoscopic examination is performed to a patient with unilateral nasal obstruction that developed from mild to severe (or complete) obstruction in months or even years, which suggests the slow growing of the tumor. Nasal endoscopy is performed very gently in order to determine the exact origin of the inverted papilloma. We found that there was no apparent origin in the nasal septum and, most of the time, the tumor originated from the ostiomeatal complex or the lateral nasal wall. We did not perform any biopsy at that moment in order not to decrease our patients' quality of life preoperatively.

The next step was the complete radiologic examination. We began with the CT scan and the key point was

\section{Table 1}

Krouse staging system for sinonasal inverted papilloma ${ }^{13}$

\begin{tabular}{|c|c|}
\hline Stage & \\
\hline I & $\begin{array}{l}\text { Tumor totally confined to the nasal cavity, without extension into the sinuses. } \\
\text { The tumor can be localized to one wall or region of the nasal cavity, or can be bulky and extensive within the nasal cavity, but must not } \\
\text { extend into the sinuses or into any extranasal compartment. } \\
\text { There must be no concurrent malignancy }\end{array}$ \\
\hline II & $\begin{array}{l}\text { Tumor involving the ostiomeatal complex, and ethmoid sinuses, and/or the medial portion of the maxillary sinus, with or without } \\
\text { involvement of the nasal cavity. } \\
\text { There must be no concurrent malignancy }\end{array}$ \\
\hline III & $\begin{array}{l}\text { Tumor involving the lateral, inferior, superior, anterior, or posterior walls of the maxillary sinus, the sphenoid sinus, and/or the frontal sinus, } \\
\text { with or without involvement of the medial portion of the maxillary sinus, the ethmoid sinuses, or the nasal cavity. } \\
\text { There must be no concurrent malignancy }\end{array}$ \\
\hline IV & $\begin{array}{l}\text { All tumors with any extranasal/extrasinus extension to involve adjacent, contiguous structures such as the orbit, the intracranial } \\
\text { compartment, or the pterygomaxill ary space. } \\
\text { All tum ors asso ciated with malignancy }\end{array}$ \\
\hline
\end{tabular}


to discover the origin of the inverted papilloma. We were looking for the small stalk representing the origin of the tumor or the hyperostosis on the walls of the paranasal sinuses in order to know exactly where to perform precise endoscopic resection.

The present study is a descriptive retrospective study, using data from a period of 15 years, between January 2000 and January 2014. The data were retrieved from the files of "Prof. Dr. Dorin Hociota" Institute for Phono-Audiology and Functional ENT Surgery, Bucharest. The study group is comprised of 162 patients $(\mathrm{n}=162)$, aged between 14 and 85 years old, patients hospitalized and treated in our clinic. The inclusion condition was the positive histological diagnosis of inverted papilloma for all of the patients. We selected our patients in stage I, II and most of stage III, knowing that the aims of inverted papilloma surgery are the complete removal of the tumor and the adjacent mucosa, the underlying bone attachment (subperiosteal dissection). We decided to leave intact the orbital periosteum if possible, to sacrifice the nasal lacrimal duct if necessary and to create a wide-open cavity. All patients had preoperative contrast-enhanced computer tomography or magnetic resonance imaging investigations.

The variables for the study were age, gender, smoker status, imagistic evaluation, tumor staging, location and extension of the tumor, recurrence, malignant transformation, surgical treatment.
The data obtained were transcribed into Microsoft Excel Worksheets, and statistically analyzed using GraphPad Software Prism 6. The statistical analysis was made using variables like "gender", "smoking status", "imagistic evaluation", "staging". For these variables, we used a percent presentation. The "age" variable is a quantitative variable with normal distribution.

\section{RESULTS}

Out of the 162 patients included in our study group, $104(64.19 \%)$ were male and $58(35.81 \%)$ were female. The patients were distributed variably across age groups, which were divided between 10-90 years into 8 groups $(10-19 \ldots 80-90)$. Most patients were included into the 50-59 years group $(35.80 \%, \mathrm{n}=58)$ and the $60-69$ years group $(25.92 \%, \mathrm{n}=42)$. The youngest patient was 14 years old and the oldest was 85 years old (Graph 1).

In our study group, the sinuses most commonly involved were the maxillary and ethmoid sinuses, affected simultaneously in 110 cases $(67.90 \%)$. The ethmoid sinus alone was affected in 45 cases $(27.77 \%)$. Singular frontal sinus and sphenoid sinus involvement was exceptional and accounted for only 7 cases in the study group, respectively 3 cases $(1.85 \%)$ for the frontal sinus and 4 cases (2.46\%) for the sphenoid (Graph 2).

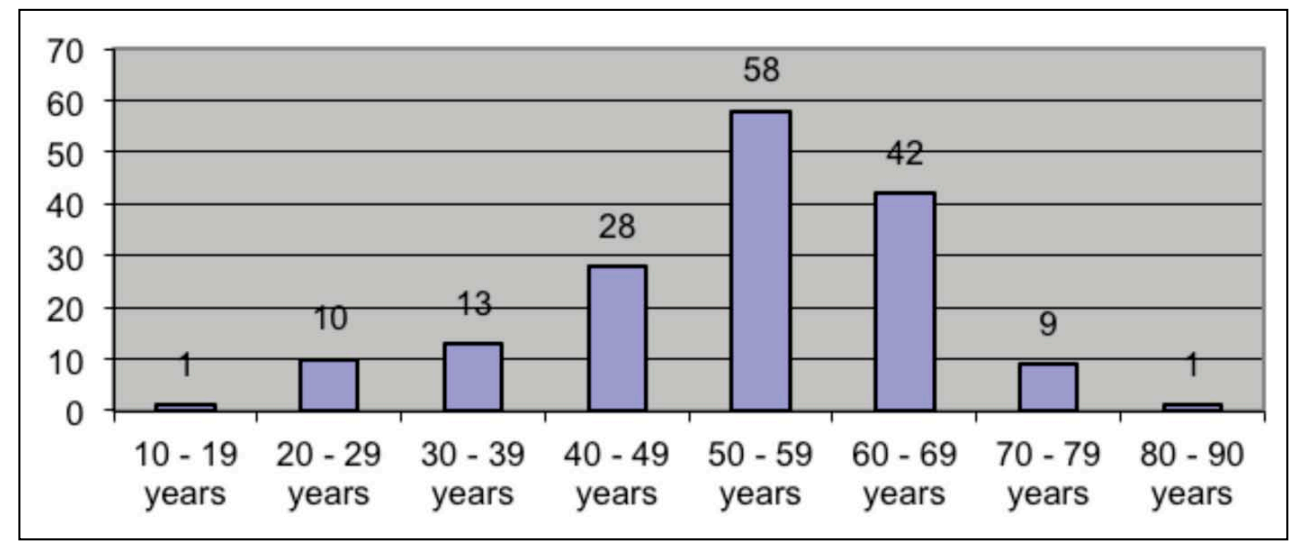

Graph 1. Distribution of patients among age groups

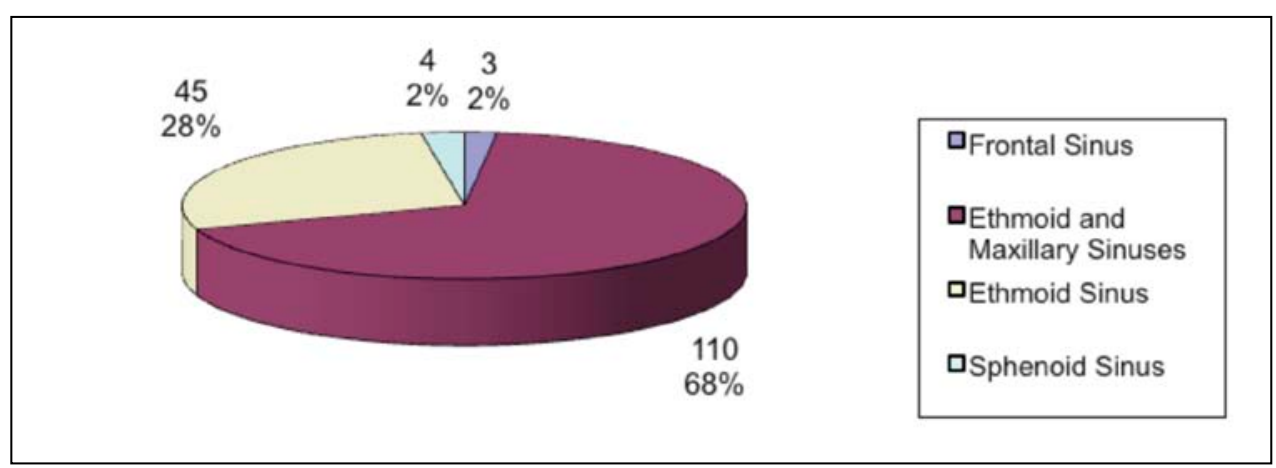

Graph 2. Sinus involvement in inverted papilloma 


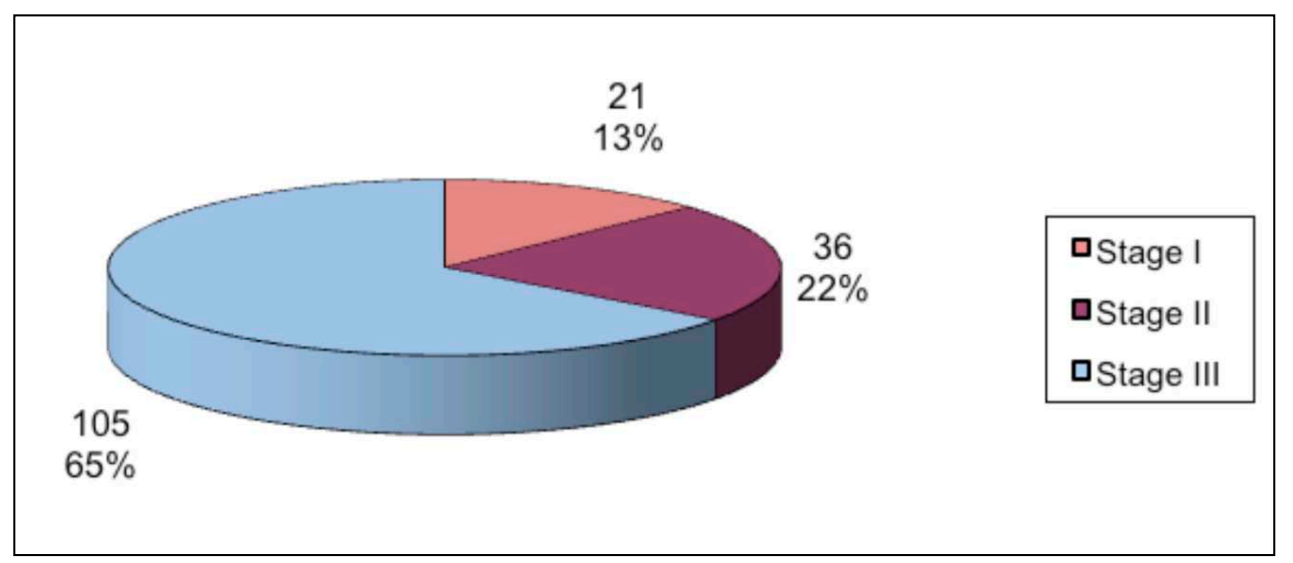

Graph 3. Distribution of patients according to Krouse's classification

The most common stage of tumor staging, according to Krouse's classification, was Stage III, with a total of 105 cases $(64.81 \%)$, followed by Stage II in 36 cases $(22.22 \%)$. The least frequent were tumors in Stage I, accounting for 21 cases (12.96\%) (Graph 3).

Treatment for all of the cases was endoscopic endonasal surgery, with different techniques, according to the Stage of the tumor. Tumors confined to the nasal cavity alone, meaning Krouse Stage I $(12.96 \%, \mathrm{n}=21)$, were treated by complete endoscopic resection of the tumor. In the case of Krouse Stage II $(22.22 \%, n=36)$, tumors mostly affecting the ethmoid sinuses, the surgical technique included endoscopic resection of the tumor and ethmoidectomy. A different approach was used for Stage III tumors, which were the most frequent in our study, and were found in $64.81 \%$ of cases $(n=105)$. In these cases we performed an endoscopic medial maxillectomy with anterior and/or posterior ethmoidectomy. In all cases, surgical specimens were sent for further histological examination.

Follow-up in our study was made by nasal endoscopy and at specific intervals, and comprises a period between 1 and 12 years. Our follow-up schedule is standardized and includes monthly controls for the first three months, four times a year for the first year, once every six months for the second and third years and once a year for the fourth and fifth years.

Recurrence of the papilloma appeared in 26 cases $(16.04 \%)$, but only for 16 patients, some of the recurrences appearing in the same patients. Most recurrences occurred in the third and fourth years after surgery. Malignancy rate was of $7.4 \%(n=12)$.

\section{DISCUSSIONS}

IP are rare tumors of the sinonasal cavity, comprising $0.5-4 \%$ of all primary nasal tumors ${ }^{14}$. There is a male predominance with approximately $3: 1$ ratio $^{1,15}$. In our study, there was clear male predominance among patients included in the study group, $64.19 \%$ of patients being males and $35.81 \%$ females.

Common sites of origin include the lateral nasal wall and medial wall of the maxillary sinus, according to most authors. Rare locations include the sphenoid sinus and frontal sinus ${ }^{1-5,9}$. In our study, the most frequent location and extension of IP was represented by involvement of both ethmoid and maxillary sinuses, in 110 cases from our series $(67.90 \%)$, concurring with most authors.

Regarding IP in our study, we used Krouse's staging system for $\mathrm{IP}^{13}$. In this system (Table 1), Stage I comprises tumors localized on one wall of the nasal cavity, without sinus involvement. This was the least frequent situation in our study (12.96\%). Stage II, according to Krouse's staging system, is represented by tumors involving the ostiomeatal complex, ethmoid sinuses and/or the medial portion of the maxillary sinus. This stage comprised $22.22 \%$ of the cases in our study. Stage III represents involvement of the maxillary sinus, of the frontal or sphenoid sinuses, with or without involvement of the ethmoid sinus, medial maxillary wall or nasal cavity. In our study, this was the most frequent situation $(64.81 \%)$. An explanation for this would be the fact that the symptoms are closely related to the sites of involvement. This being said, tumors developing in the maxillary sinus will have to grow and extend to different close regions before expressing any discomfort to the patient, thus delaying the diagnosis. Common symptoms of IP are progressive unilateral nasal obstruction, blood mixed nasal discharge, headache, frequent throat clearing, sometimes epiphora or symptoms of sinusitis ${ }^{4,5,16}$.

Clinical and paraclinical assessment of IP includes nasal endoscopy with anatomic macroscopic description of the tumor, imaging studies and also biopsy. Contrast-enhanced CT was used in most cases in our study. Unilateral lesions, with local hyperostosis, sometimes with intra-tumoral calcifications, bowing of the sinuses and sometimes erosion, are suggestive for 
$\mathrm{IP}^{9,10,17}$. MRI is sometimes helpful in preoperative diagnosis, showing a columnar pattern, with a cerebriform pattern. Occasionally, central necrosis of the tumor can appear, suggesting coexisting carcinoma ${ }^{1,12}$.

Treatment of IP is well debated among rhinologists, with different opinions about the best treatment option. Surgical principles dictate wide excision of the tumor with normal surrounding tissue, in order to prevent recurrence. Classical open techniques, such as lateral rhinotomy or midfacial degloving with medial maxillectomy, are rarely used today, especially for initial stages of papillomas. Nowadays, endoscopic sinus surgery is the best treatment option for initial and even more advanced papillomas. Contraindications of endoscopic surgery include the presence of squamous cell carcinoma, intradural or orbital extension ${ }^{18-21}$. In our study, most cases, staged as Stage III Krouse, were treated by endoscopic surgery by medial maxillectomy and sometimes followed by anterior and posterior ethmoidectomy. Some literature reviews show a smaller recurrence rate following endoscopic surgery for IP, reaching values from 3 to $17 \%$, whilst recurrence after open techniques is comprised between 18$24 \%^{1,22-24}$. In our study, recurrence was of $16.04 \%$, mostly occurring in the third or fourth years after surgery, and there was a tendency that patients who had a recurrence would more likely have another one. This could probably be explained by the possible presence of intracellular HPV DNA, which is supposed to be related to higher chances of recurrence and malignant transformation ${ }^{1,25}$. Some authors, on the other hand, proved that there are no significant statistical data in order to combine HPV DNA to frequent recurrence of $\mathrm{IP}^{26}$.

Besides surgery, other treatment types have been suggested, like medication and radiotherapy. Therapy including Interferon and other antiviral drugs has been suggested, like intralesional therapy with Cidofovir, for patients with multiple recurrences or advanced disease. They are still under investigation ${ }^{27-29}$.

\section{CONCLUSIONS}

Endoscopic endonasal surgery for IP is currently the standard treatment for sinonasal IP, especially IP staged from I-III according to the staging of Krouse. Endoscopic endonasal surgery is considered to have less recurrence than more difficult, more aggressive open surgical techniques. Recurrence after endoscopic surgery is of $16.04 \%$, according to our study.

In order to have the best results in the treatment of IP, some details are crucial: the complete and correct diagnosis, precise surgical technique and rigorous follow-up. Complete diagnosis is comprised of nasal endoscopy, imaging studies (CT, MRI) and histologi- cal confirmation from biopsy or surgical specimen. The surgical technique has to be tailored to tumoral stage and extensions. For most IP, endoscopic medial maxillectomy, combined with ethmoidectomy, is the treatment of choice. Follow-up through nasal endoscopy has to be scheduled and rigorously followed.

In selected cases, surgical techniques could be associated to other types of therapy, like antiviral and antiangiogenic agents.

\section{REFERENCES}

1. Thapa N. - Diagnosis and treatment of sinonasal inverted papilloma. Nepalese Journal of ENT Head and neck Surgery, 2010;1(1):30-33.

2. Cho H.J., Kim J.K., Kim K. - Endoscopic surgery for inverted papilloma originating from the sphenoid sinus and related clinical characteristics. Acta Otolaryngol., 2008;128:1120-1125.

3. Peters B.W., O'Reilly R.C., Wilcox J. - Inverted papilloma isolated to the sphenoid sinus. BMJ Case Reports, 2009;10:1136.

4. de Filippis C., Marioni G., Tregnaghi A., Marino F., Gaio E., Staffieri A. Primary inverted papilloma of the middle ear and mastoid. Otol Neurotol., 2002;23:555-559

5. Phillips P.P., Gustafson R.O., Facer G.W. - The clinical behavior of inverting papilloma of the nose and paranasal sinuses: report of 112 cases and review of literature. Laryngoscope, 1990;100:463-69.

6. Gaffey M.J., Frierson H.F., Weiss L.M. - Human papillomavirus and EpsteinBarr virus in Schneiderian papillomas. An in situ hybridization and polymerase chain reaction study. Am J Clin Pathol., 1996;106(4):475-82.

7. Hwang C.S., Yang H.S. - Detection of human papillomavirus (HPV) in sinonasal inverted papillomas using polymerase chain reaction (PCR). Am J Rhinol., 1998;12(5):363-6.

8. Ringertz N. - Pathology of malignant tumors arising in the nasal and paranasal cavities and maxilla. Acta Otolaryngol (Stockh)., 1938;27(suppl):31-42.

9. Dammann F., Pereira P.M., Laniado M. - Inverted papilloma of the nasal cavity and the paranasal sinuses: using CT for primary diagnosis and followup. Am J Roentgenol., 1999;172:543-548.

10. Woodruff W.W., Vrabec D.P. - Inverted papilloma of the nasal vault and paranasal sinuses: spectrum of CT findings. Am J Roentgenol., 1994;162:419 435.

11. Jeon T.Y., Kim H.J., Chung S.K. - Sinonasal inverted papilloma: value of convoluted cerebriform pattern on MR imaging. American Journal of Neuroradiology, 2008;29:1556-1560.

12. Ojiri H., Ujita M., Tada S. - Potentially distinctive features of sinonasal inverter papilloma on MR imaging. Am J Roentgenol., 2000;175:465-68.

13. Krouse J.H. - Development of a Staging System for Inverted Papilloma. The Laryngoscope, 2000;110:965-968.

14. Sadeghi N. - Sinonasal papillomas, treatment. 2002. E-medicine. www.e-medicine.com/ent/topic529

15. Sauter A., Matharu R., Horman K. - Current advances in the basic research and clinical management of sinonasal inverted papilloma (review). Oncol Rep., 2007;17:495-504.

16. Visvanathan V., Wallace H., Chumas P. - An unusual presentation of inverted papilloma: case report and literature review, 2009;3:1-4.

17. Lund V.J., Lloyd G.A. - Radiological changes associated with inverted papilloma of the nose and paranasal sinuses. Br J Radiol., 1984:57:455-61.

18. Lee T.J., Huang S.F., Lee L.A. - Endoscopic surgery for recurrent inverted papilloma. Laryngoscope, 2004;114:106-112.

19. Sauter N.B., Cannady S.B., Citardi M.J., Roh H.J., Batra P.S. - Comparison of open vs. endoscopic resection of inverted papilloma. Am J Rhinol., 2007;21(3):320-323

20. Jameson M.K., Kountakis S.E. - Endoscopic management of extensive inverted papilloma. Am J Rhinol., 2005;19:446-451. 
21. Von Buchald C., Larsen A.S. - Endoscopic surgery of inverted papillomas under image guidance: a prospective study of 42 consecutive cases at a Danish university clinic. Otolaryngol Head Neck Surg., 2005;132(4):602-607.

22. Pasquini E., Sclaretta V., Fameti G. - Inverted papilloma: report of 89 cases. Am J Otolaryngol., 2004;25:178-85.

23. Lawson W., Kaufman M.R., Biller H.F. - Treatment outcomes in the management of inverted papilloma: an analysis on 160 cases. Laryngoscope, 2003;113:1548-56.

24. Kraft M., Kaufmann T., Holzmann D. - Long-term results of endonasal sinus surgery in sinonasal papillomas. Laryngoscope, 2003;113:1541-47.

25. Ogura H., Fukushime K., Watanabe S. - A high prevalence of human papillomavirus DNA in recurrent nasal papillomas. J Med Microbiol., 1996;45(3):162-6.
26. Jenko K., Kocjan B., Zidar N., Poijak M., Strojan P., Zargi M., Blatnik O., Gale N. - In inverted papillomas HPV more likely represents incidental colonization than an etilogical factor. Virchows Arch., 2011;459(5):529-38.

27. Nakano T., Cho H., Sugita M., Shiotani H., Matsumoto Y., Yamada K. - A case of a nasal cavity inverted papilloma treated with a local injection of 囚-interferon. Practica Otologica., 1999;92(11):1201-1204.

28. Petersen B.L., Buchwald C., Gerstoft J., Bretlau P., Lindberg H. - An aggressive and invasive growth of juvenile papillomas involving the total respiratory tract. J Laryngol Otol., 1998 Nov;112(11):1101-4.

29. Wassef S.N., Batra P.S., Barnett S. - Skull Base Inverted Papilloma: A Comprehensive Review. ISRN Surgery, 2012;2012:175903. doi:10.5402/2012/175903. 